UDC 539.4

\title{
Theoretical-Experimental Model for Predicting Crack Growth Rate in Structural Alloys under Combined Action of Fatigue and Creep
}

\author{
V. V. Pokrovskii, ${ }^{a}$ V. G. Sidyachenko, ${ }^{a}$ V. N. Ezhov, ${ }^{a}$ and S. B. Kulishov ${ }^{b}$ \\ a Pisarenko Institute of Problems of Strength, National Academy of Sciences of Ukraine, \\ Kiev, Ukraine \\ ${ }^{\mathrm{b}}$ Zorya-Mashproekt (State Research and Production Center for Problems of Gas Turbine \\ Construction), Nikolaev, Ukraine
}

Holding periods of 300 and $3600 \mathrm{~s}$ in a trapezoidal load cycle are shown to increase the crack growth rate dozens of times for alloy EP962 and several-fold for alloy EP742 at a temperature of $973 \mathrm{~K} . \mathrm{It}$ is demonstrated that in the presence of the first portion on the creep crack growth diagram, whereon the crack growth rate decreases, the crack growth kinetics for a trapezoidal load cycle can be predicted using the hypothesis of the linear summation of fatigue and creep crack growth rates provided that the peculiarities of the first portion of the creep crack growth diagram are taken into account. Empirical approaches are proposed for determining the mean crack velocity in the first portion of the creep crack growth diagram.

Keywords: crack, trapezoidal cycle, creep-fatigue interaction.

Introduction. Analysis of the state of the problem revealed that at present the issues concerning prediction of the crack growth kinetics under hightemperature fatigue and long-term static loading are the best elaborated ones [1-3]. Less well understood are the problems of creep crack development in materials that fracture in a brittle mode [4-6]. Also, there is a lack of experimental data and theoretical generalization on crack propagation for a trapezoidal load cycle with various hold periods under maximum load $[7,8]$. The majority of researchers in this case restrict themselves to the use of the linear damage summation hypothesis based on the independent damaging effect of fatigue and creep $[2,9]$. Sometimes, they determine the material susceptibility to a certain mode of fracture (fatigue or time-dependent) and, on this basis, choose the fracture mechanics parameters to describe experimental crack growth rate data $[10,11]$. It is noteworthy that such approaches do not always yield satisfactory results and require further experimental and physical substantiation.

Earlier [12, 13], on the basis of the experimental data obtained and calculations performed, it was shown that the most commonly used approaches give no way of predicting the crack growth kinetics in the alloys under study to sufficient accuracy. In this paper, we will discuss the methods proposed for the solution of the above problem.

Research Task Statement. High-temperature nickel-based alloys ÉP742 and ÉP962 for aircraft applications were used as model materials. Their mechanical properties and other characteristics as well as the experimental procedure were detailed earlier in $[12,14]$. The materials under study are used to manufacture long-life aircraft gas-turbine engine (AGTE) disks. The service conditions (flight cycle) involve operation of the material under cyclic loading with long hold periods at maximum load in a cycle. The solution of this problem makes it 
possible to determine the number of cycles of subcritical crack growth, which should be taken into account when determining the terms and amount of scheduled maintenance sessions depending on the technical condition, and to preclude catastrophic failure of AGTE disks.

To construct a model for predicting the influence of hold time on the crack growth rate, it is necessary to perform the following experimental investigations:

(i) to study the crack growth behavior under cyclic and long-term static loading;

(ii) to study the crack growth rate (CGR) under loading with a trapezoidal cycle and with holding periods of 300 and $3600 \mathrm{~s}$ at maximum load.

Theoretical-Experimental Model. To construct a model we used the experimental CGR data obtained at a constant load with a trapezoidal and triangular loading cycle shown in Figs. 1-4.

Since the duration of the first portion of the CCG diagram at initial $K_{\text {in }}$ values is much longer than that of the flight cycle (by a factor of 10 and more), we propose that the equation of the linear crack growth rate summation should involve the value of the mean CCG rate in the first portion $\dot{a}_{m}$ instead of the conventionally used steady-state CCG rate (Fig. 1).

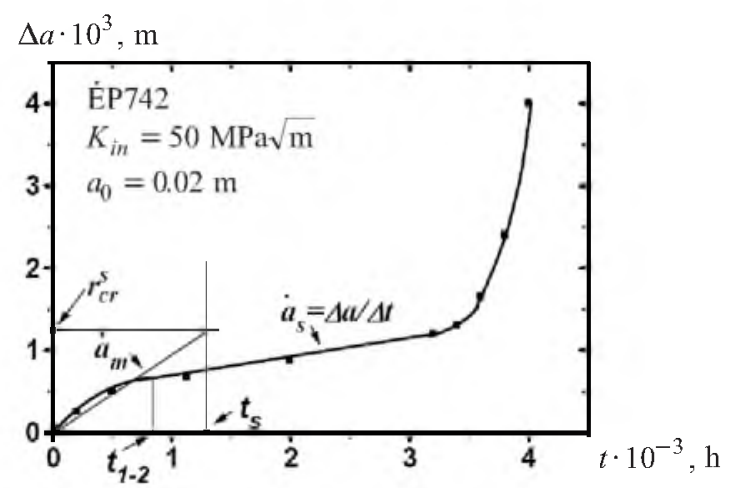

Fig. 1. Crack length increment $\Delta a$ vs. loading time $t$.

Moreover, during fatigue crack growth (triangular cycle), a transcrystalline mode of fracture is most often realized in the alloys under study, whereas during crack growth under creep conditions an intercrystalline mode of fracture is observed $[2,18]$. In this case, the presence of the first portion depends on the crack initiation technique. In the case of an initial fatigue crack present, the first portion did exist on the CCG diagram constructed in the coordinates $\Delta a-t$. This was associated with a change in the fracture mechanism in transition from a fatigue crack to a creep one. In studies of the creep crack growth directly from a stress concentrator, the crack propagated at a constant rate, i.e., according to the mechanism characteristic of the second portion with the intergranular fracture mechanism predominant. Since the trapezoidal cycle represents combined loading (cyclic plus static), the crack growth during holding periods is most likely to occur by the mechanisms responsible for the first portion of the CCG diagram. In view of the aforesaid, we write the hypothesis of the linear rate summation in the following form: 


$$
\left(\frac{d a}{d N}\right)_{C F}=B\left(K_{\max }\right)^{m}+\dot{a}_{m} t_{h}
$$

where $\left(\frac{d a}{d N}\right)_{C F}$ and $\dot{a}_{m}$ are the crack growth rate for a trapezoidal loading cycle and the mean rate in the first portion of the CCG, respectively, $B$ and $m$ are the Paris equation coefficients determined from the CGR diagram for a triangular loading cycle, and $t_{h}$ is the hold time.

The most reliable method for the $\dot{a}_{m}$ evaluation is the experimental determination of the time $\left(t_{1-2}\right)$ when the crack reaches the portion of constant growth rate as well as the crack increment $\Delta a$ corresponding to this instant of time (Fig. 2). However, performing such experiments is a cumbersome process due to certain difficulties involved in visual observation of the creep crack increment, particularly at the initial stage of the experiment, and the necessity of following the procedure as described in $[14,19]$. From Fig. 1 we notice that the crack increment in the first portion is insignificant and is approximately $0.2-$ $0.7 \mathrm{~mm}$. The crack in this case can grow not only on the surface but also in the bulk of the specimen and change its front curvature.
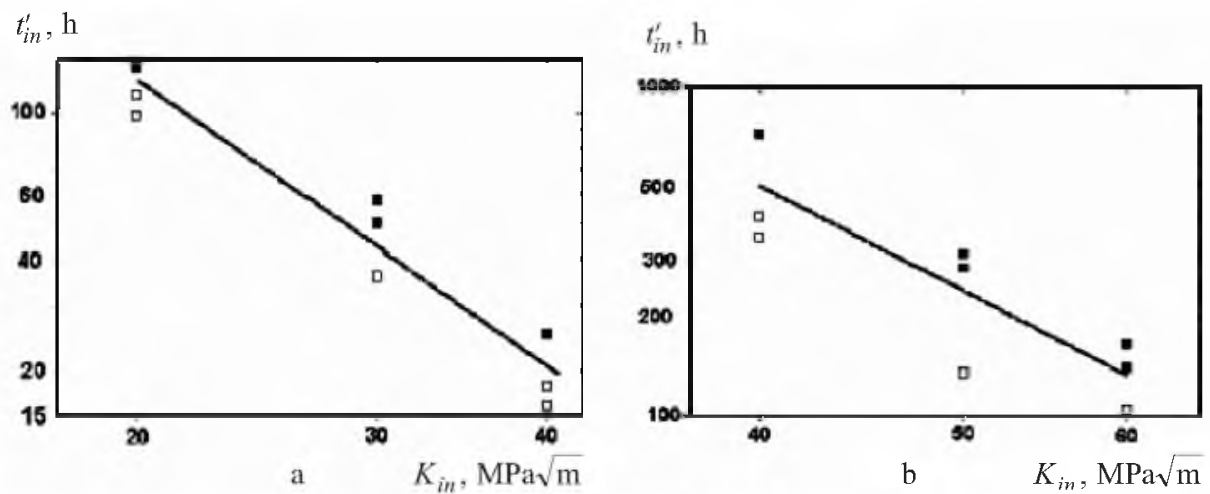

Fig. 2. Relation $t_{i n}^{\prime}-K_{\text {in }}$ for alloys ÉP962 (a) and ÉP742 (b)

In accordance with [19], $t_{\text {in }}^{\prime}$ is the time from the instant of load application to the crack increment by $0.2 \mathrm{~mm}$, which can be determined approximately using the technique described earlier [14]. In addition, this magnitude of the increment corresponds to the onset of the steady-state growth of the creep crack, as noted by many researchers $[4,16,19]$.

The dependence of the conditional incubation period $t_{\text {in }}^{\prime}$ on the stress intensity factor (SIF) $K_{i n}$ is illustrated in Fig. 2 and represented by the empirical relation

$$
t_{i n}^{\prime}=\frac{C}{K^{p}}
$$

Based on definition of the conditional incubation period, we determine the mean crack growth rate during holding periods by dividing the crack increment, 
$\Delta a \approx 0.2 \mathrm{~mm}$, by the time, $t_{i n}^{\prime}$, within which this increment occurred. Then, the formula for the crack growth rate in a trapezoidal loading cycle takes the form

$$
\left(\frac{d a}{d N}\right)_{C F}=B\left(K_{\max }\right)^{m}+\frac{0.2 \cdot 10^{-3}}{t_{\text {in }}^{\prime}} \iota_{h} .
$$

From the diagrams in Fig. 3 it follows that for alloy ÉP962 a satisfactory agreement is observed between the calculation by formula (3) and experimental data obtained for the hold times $t_{h}=300$ and $3600 \mathrm{~s}$. However, for alloy ÉP742 the calculation by the modified linear hypothesis (3) fails to give an acceptable CGR estimate for a holding period of $300 \mathrm{~s}$.
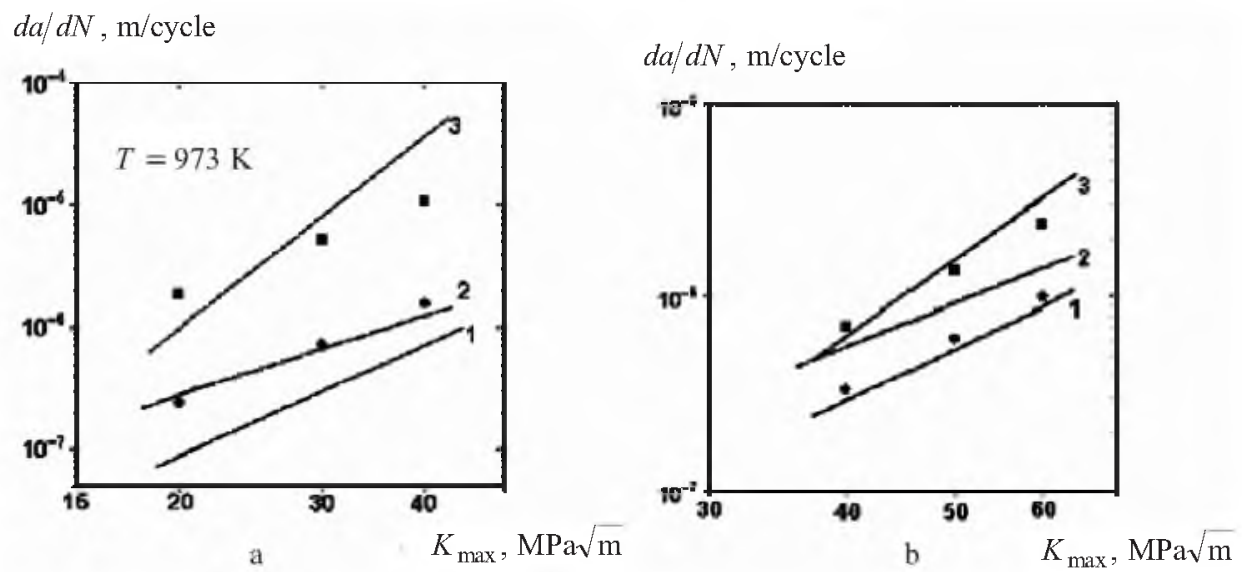

Fig. 3. Experimental (lines) and calculated (dots) functions $(d a / d N)-K_{\max }$ for alloys EP962 (a) and EP742 (b) under cyclic loading with the hold time $t_{h}$ : (I) $f=0.5 \mathrm{~Hz}, t_{h}=0$; (2) $t_{h}=300 \mathrm{~s}$; (3) $t_{h}=3600 \mathrm{~s}$; (ם) and ( calculation by (3) with hold times $t_{h}=3600$ and $300 \mathrm{~s}$, respectively.

To refine the linear hypothesis (1), we analyze the stress state at the tip of a quasi-static creep crack in order to determine the mean crack velocity $\dot{a}_{m}$ in the initial portion of the creep crack growth diagram. When a cracked specimen is loaded with a constant load, there occur a crack-tip stress relaxation and the development of a creep zone whose size is defined by the following relation [11]:

$$
r_{c r}=\left[\frac{1}{2 \pi}\left(\frac{(n+1)^{2}}{2 n \alpha_{n}^{n+1}} E A\right)^{2 /(n-1)} F_{c r}(\theta)\right] K^{2} t^{2 /(n-1)},
$$

where $K$ is the SIF, $E$ is the elastic modulus, $t$ is the current time, $F_{c r}(\theta)$ is the geometrical factor, $\alpha_{n}^{n+1} \approx 0.69$, and $n$ and $A$ are the values of the coefficients in the equation that describes the minimum creep rate for a smooth specimen:

$$
\dot{\varepsilon}=A \sigma^{n}
$$


Simultaneously, the process of damage accumulation starts in the material at the crack tip (formation of pores, intergranular cracking, etc.) resulting in the crack increment and ultimately in a decrease in the initial creep zone size (4). Thus, the crack-tip stresses decrease due to relaxation and, at the same time, increase owing to the crack increment and to the fact that the crack tip approaches the creep zone boundary. In the first portion of the CCG diagram the processes described above are of transient nature, and as time passes the stabilization of both the CGR and the rate of displacement along the force action line takes place (Figs. 1 and 3). Thus, the condition for the crack to reach the portion of constant velocity is the equality of the CCG rate and the creep zone extension rate at the crack tip:

$$
\dot{a}_{s}=\dot{r}_{c r} .
$$

Differentiation of relation (4) with respect to time gives an estimate of the time $\left(t_{s}\right)$ at the expiration of which the condition (6) is satisfied

$$
t_{s}=\left(\frac{2 D}{n-1}\right)^{\frac{n-1}{n-3}}\left(\frac{K^{2}}{\dot{a}_{s}}\right)^{\frac{n-1}{n-3}} \text {, }
$$

where $D$ is the square-bracketed expression in (4) and $\dot{a}_{s}$ is the CGR in the second portion of the CCG diagram (Fig. 1). Other notations are as described above.

The size of the crack-tip creep zone at the time $t_{s}$ is given by

$$
r_{c r}^{s}=D K^{2}\left(\frac{2 D}{n-1}\right)^{\frac{2}{n-3}}\left(\frac{K^{2}}{\dot{a}_{s}}\right)^{\frac{2}{n-3}} .
$$

Assuming that the crack, before reaching the second portion, would extend by the value corresponding to the creep zone size $r_{c r}^{s}$ within the time $t_{s}$, we estimate its mean rate in the first portion as

$$
\dot{a}_{m}=\frac{r_{c r}^{s}}{t_{s}} .
$$

In order to use relation (1) modified in view of (4)-(9), we plotted kinetic creep crack growth diagrams in the coordinates $\dot{a}_{s}-K$. The SIF value in the linear portion varies but slightly (due to a constant load and small increment in the crack length). relation:

The diagram in the coordinates $\dot{a}_{s}-K$ is described by the following

$$
\dot{a}_{s}=B(K)^{q} .
$$


The $\dot{a}_{s}$ values were calculated by taking into account only the second (linear) portion of each diagram obtained at a constant load $\left(P_{i}=\right.$ const $)$ and the SIF value corresponding to this load.

Substitution of expressions (7) and (8) into (9) with subsequent rearrangement gives a relation between the mean CCG rate in the initial portion, $\dot{a}_{m}$, and the rate in the second portion:

$$
\dot{a}_{m}=\frac{n-1}{2} \dot{a}_{s}
$$

Verification of applicability of the method for determining $\dot{a}_{m}$ by formulas (4)-(11) to modify Eq. (1) is provided in Fig. 4. A satisfactory agreement between the prediction and experiment is observed at a hold time $t_{h}=3600 \mathrm{~s}$, i.e., during long holding periods, when the processes of the crack-tip stress redistribution become less pronounced [as the fulfillment of condition (6) is approached].
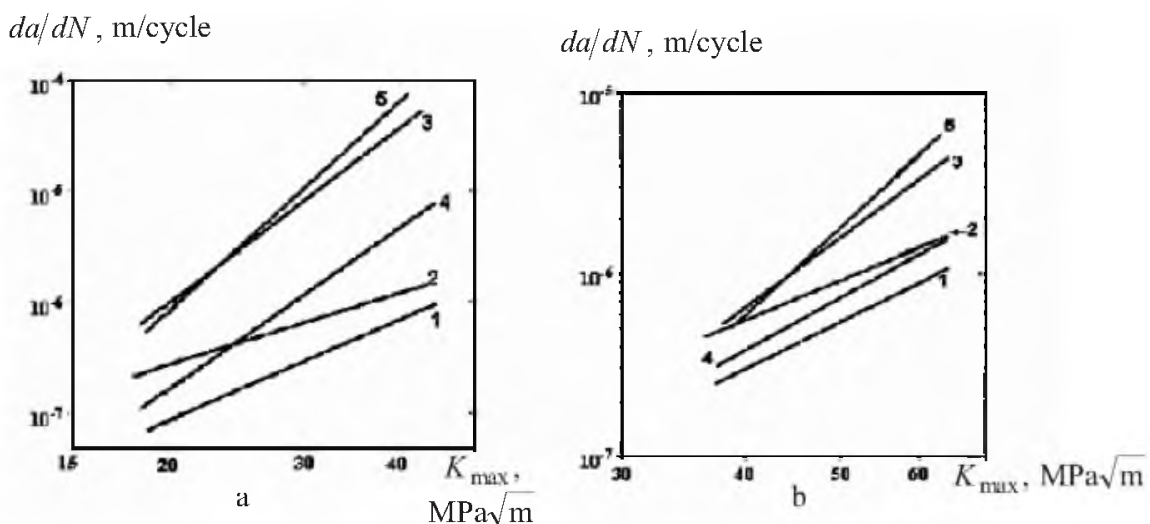

Fig. 4. Experimental $(I-3)$ and calculated $(4,5)$ functions $d a / d N-K_{\max }$ for alloys ÉP962 (a) and ÉP742 (b) under cyclic loading with a hold time $t_{h}:$ (I) $t_{h}=0$; (2) $t_{h}=300 \mathrm{~s}$; (3) $t_{h}=3600 \mathrm{~s}$; (4, 5) calculation by (1) in view of (4)-(9) with $t_{h}=300$ and $3600 \mathrm{~s}$, respectively.

However, at $t_{h}=300 \mathrm{~s}$ the prediction by relation (1) falls short of being encouraging owing to the concurrent processes of the stress relaxation due to creep and stress increasing through the crack extension increment, which are far from the stabilization condition (6) during short holding periods.

\section{Conclusions}

1. Based on generalization of experimental data, we proposed a modification of the linear hypothesis of the crack growth rate summation under combined action of fatigue and creep. It involves the use of the value of the mean creep crack growth rate in the initial portion of the crack growth diagram instead of the current creep crack growth rate.

2. The calculation of the crack growth rate for a trapezoidal cycle using the modified linear hypothesis of the crack growth rate summation has revealed that in the region of low SIF values (closer to the threshold ones) the application of the second method for determining the mean creep crack growth rate yields a 
somewhat overestimated value as compared to experimental one, whereas the use of the first method gives an underestimated value. When the calculations by the proposed methods are performed for the region of high SIF values (closer to the critical ones), just an opposite tendency is observed. For this reason, it is proposed that the creep crack growth rate in the first portion of the crack growth diagram should be evaluated by two methods and that the calculated results that predict higher crack growth rates should be used in the modified hypothesis of the linear rate summation.

3. As a result of the stress state analysis in the vicinity of the crack tip, we have derived an expression relating the mean crack growth rate in the initial portion to the steady-state rate in the second portion. The relation obtained depends solely on the material properties and is invariant to the applied external load.

1. S. Taira and R. Otani, The Theory of High-Temperature Strength of Materials [Russian translation], Metallurgiya, Moscow (1986).

2. R. P. Skelton (Ed.), High-Temperature Fatigue of Materials [Russian translation], Metallurgiya, Moscow (1988).

3. K. J. Miller, Creep and Fracture [Russian translation], Metallurgiya, Moscow (1986).

4. K.-H. Schwalbe, R. H. Ainsworth, A. Saxena, and T. Yokobori, "Recommendation for a modification of ASTM E 1457 to include creep-brittle materials," Eng. Fract. Mech., 62, 123-142 (1999).

5. A. Saxena, D. E. Hall, and D. L. McDowell, "Assessment of the deflection rate for analyzing creep crack growth data," Eng. Fract. Mech., 62, 111-122 (1999).

6. A. T. Yokobori, Jr., "Difference in the creep and creep crack growth behavior between creep ductile and creep brittle materials," Eng. Fract. Mech., 62, 61-78 (1999).

7. A. Saxena, R. S. Williams, and T. T. Shih, "A model for representing and predicting the influence of hold time on fatigue crack growth behavior at elevated temperature," in: Fracture Mechanics: Thirteenth Conf., ASTM STP 743 (1981), pp. 86-99.

8. Kee Bong Yoon, A. Saxena, and P. K. Liaw, "Characterization of creepfatigue behavior under trapezoidal waveshape using $C_{t}$-parameter," Int. $J$. Fract, 59, 95-114 (1993).

9. J. P. Perdon and A. Pineau, "Effect of hold times on the elevated temperature fatigue crack growth behaviour of Inconel 718 alloy," in: Proc. of the Int. Conf. on Advances in Fracture Research, ICF 5 (Cannes, France, 1981), Oxford (1981), p. 2385.

10. S. Mall, E. A. Staubs, and T. Nicholas, "Investigation of creep/fatigue interaction on crack growth in a titanium aluminide alloy," J. Eng. Mater. Technol., 112, 435-441 (1990).

11. H. Riedel, Fracture at High Temperatures, Springer-Verlag, Berlin (1987). 
12. V. V. Pokrovskii, V. N. Ezhov, and V. G. Sidyachenko, "Prediction of crack growth rate in alloys ÉP742 and ÉP962 under combined action of cyclic and static loads at a temperature of 973 K," Vestnik KPI. Ser. Mashinostroenie, 41, 221-227 (2001).

13. V. V. Pokrovskii, V. N. Ezhov, and V. G. Sidyachenko, "Prediction of the crack growth rate in disc materials taking into account loading conditions," in: Proc. Int. Conf. on Life Assessment and Management for Structural Components [in Russian], Vol. 2, National Academy of Sciences of Ukraine, Institute of Problems of Strength, Kiev (2000), pp. 851-856.

14. V. V. Pokrovskii, V. N. Ezhov, and V. G. Sidyachenko, "Special features of creep-crack propagation in refractory nickel alloys under static loading," Strength Mater., 33, No. 5, 438-446 (2001).

15. A. Saxena and J. D. Landes, "Characterization of creep crack growth in metals," in: Proc. ICF6, New Delhi (1984), pp. 3977-3987.

16. O. Kwon, K. M. Nikbin, G. A. Webster, and K. V. Jata, "Crack growth in the presence of limited creep deformation," Eng. Fract. Mech., 62, 33-46 (1999).

17. M. Tabuchi, K. Kubo, K. Yagi, et al., "Results of a Japanese Round Robin on creep crack growth evaluation methods for Ni-base superalloys," Ibid, 47-60 (1999).

18. A. Fuji, M. Tabuchi, A. T. Yokobori, et al., "Influence of notch shape and geometry during creep crack growth testing of TiAl intermetallic compounds," Ibid, 23-32 (1999).

19. V. A. Vainshtok, M. V. Baumshtein, I. A. Makovetskaya, and I. A. Man'ko, "Kinetics and mechanisms of creep crack growth in a creep-resisting stee1," Strength Mater., 17, No. 6, 734-739 (1985). 